\title{
Expression of Heparin-binding Epidermal Growth Factor-like Growth Factor (HB-EGF) in Human Renal Cell Carcinoma
}

\author{
Hiroshi Masuda ${ }^{1,2^{*}}$, Yoshitatsu Fukabori ${ }^{2}$, Katsuya Nakano ${ }^{2}$, Mikio Kobayashi $^{2}$ and Hidetoshi Yamanaka $^{2}$ \\ ${ }^{1}$ Department of Urology, Teikyo University Chiba Medical Center, Japan \\ ${ }^{2}$ Department of Urology, The Renal Cell Carcinoma Group of Gunma University School of Medicine, Japan
}

"Corresponding author: Dr. Hiroshi Masuda, Department of Urology, Teikyo University Chiba Medical Center, Chiba, Japan, Fax: +81-436-61-4773; Tel: +81-436-62-1211; E-mail: hrsmasuda@yahoo.co.jp

Rec date: May 05, 2016; Acc date: June 01, 2016; Pub date: June 06, 2016

Copyright: (C) 2015 Masuda $\mathrm{H}$, et al. This is an open-access article distributed under the terms of the Creative Commons Attribution License, which permits unrestricted use, distribution, and reproduction in any medium, provided the original author and source are credited.

\begin{abstract}
Background: The levels of expression of Heparin binding-epidermal growth factor like growth factor (HB-EGF) mRNA in tumor tissues and normal tissues of the excised kidney were compared in order to clarify the role of HBEGF in renal cell carcinoma (RCC) derived from the proximal tubule.

Method: Normal and tumor tissues were collected from surgical specimens of 16 cases pathologically diagnosed with RCC. Total RNA was extracted from these samples, and the level of expression of HB-EGF mRNA was measured by real-time quantitative PCR using a TaqMan probe after reverse transcription. Glyceraldehyde phosphate dehydrogenase (GAPDH) was used as an internal standard. The expression levels of HB-EGF mRNA in normal and tumor tissues of the same case were compared, and statistical analysis was performed to evaluate the association between the expression level and various clinical-pathological factors in RCC.
\end{abstract}

Results: Expression of HB-EGF mRNA was detected in $82 \%(13 / 16)$ of the normal tissues and $63 \%(10 / 16)$ of the tumor tissues. The expression level in the normal tissues was significantly 7 -fold higher than that in the tumor tissues. No significant association was detected between the expression of HB-EGF mRNA and the clinical stage or prognosis of RCC. However, the pathological findings indicated that negative expression of ratio of HB-EGF was higher in RCC with more-advanced malignant progression.

Conclusion: Our results indicated that it was unlikely that HB-EGF might play a role in determining the aggressiveness or clinical features in RCC. However, the decreased expression of HB-EGF mRNA in RCC tissues indicates that tumorigenesis of RCC may disrupt the normal regulatory system of HB-EGF.

\section{Keywords:}

\section{Introduction}

Heparin-binding epidermal growth factor like growth factor (HBEGF) belongs to the EGF superfamily and is a growth factor that binds to the EGF receptor. HB-EGF is synthesized as a precursor that consists of 208 amino acids. After it was expressed as a type (proHBEGF) that associated with the cell membrane surface, it was discharged to the extracellular space as a free type (sHB-EGF) that consisted of 87 amino acids as a result of processing by proteases $[1,2]$.

HB-EGF has been detected in heart, lung, kidney and many other organs [3]. HB-EGF is ubiquitously present in the epithelial cells of the proximal tubules and the arterial smooth muscle cells, while the expression of HB-EGF is not detected in other parts of the kidney, including the glomeruli [4]. HB-EGF is an epithelial, fibroblast and smooth muscle mitogen [5], and its known biological activities together with its stromal location in the prostate suggest a role for this molecule in benign prostatic hyperplasia (BPH) [6]. The cell surface form of HB-EGF also serves as the high affinity receptor for diphtheria toxin in human cells, mediating toxin entry and cell killing via a receptor-mediated endocytotic mechanism [7]. It has been reported that the expression of HB-EGF is highly expressed in hepatocellular carcinoma (HCC) tissues but not in normal liver [8]. However, a few reports have shown that HB-EGF is observed in urological diseases. Moreover, no report on the expression and the role of HB-EGF in renal cell carcinoma (RCC) has been published thus far.

Real-time PCR is a rapid and highly sensitive method for determining the mRNA level of HB-EGF. In addition, this method allows for the analysis of numerous samples within 3 hours ( 40 cycles). This technique can generate yes-or-no results much faster than Northern blotting, ribonuclease protection assays or various quantitative reverse transcription-polymerase chain reaction (RTPCR) techniques $[9,10]$.

We compared the expression of HB-EGF mRNA in tumor and normal tissues from 16 RCC patients in order to clarify the role of HBEGF in RCC.

\section{Material and Methods}

\section{Patients and specimens}

We examined 16 patients (10 men and 6 women) who underwent radical nephrectomy at Gunma University Hospital. All tumors in the 16 patients were diagnosed histologically as RCC. We obtained normal and tumor tissues from the surgical specimens. The tissues were taken 
Citation: Masuda H, Fukabori Y, Nakano K, Kobayashi M, Yamanaka H (2015) Expression of Heparin-binding Epidermal Growth Factor-like Growth Factor (HB-EGF) in Human Renal Cell Carcinoma . J Integr Oncol 5: 171. doi:10.4172/2329-6771.1000171

Page 2 of 4

after informed consent was obtained from the patient or members of the patient's family. The mean patient age was 63 years (range 38 to 76). The mean follow-up was 49 months (range 42 to 57 ).

\section{Sample tissues}

Normal and tumor kidney tissues obtained from the operation were immediately frozen in liquid nitrogen and stored until RNA extraction. These samples were stored at -80 .

\section{RNA extraction}

The tissues were crushed with a mallet while frozen, and were homogenized in TRIzol reagent (Life Technologies, Maryland, USA) using an ultrasonic cell disrupter (Wakenyaku Co., Ltd., Kyoto, Japan). Total RNA was extracted according to the manufacturer's instructions (Life Technologies, Maryland, USA).

\section{The design of the TaqMan probe and PCR primer}

A TaqMan probe and PCR primers for HB-EGF were designed using Primer Express, version 1.0 (Perkin-Elmer Applied Bio systems Inc., Foster City, CA, USA), and they were purchased from a commercial company. The internal probe was labeled at the 5 ' end with the reporter dye FAM (6-carboxy-fluorescein), and at the 3' end with the quencher dye TAMRA (6-carboxy-tetramethyl-rhodamine), and was phosphate-blocked at the 3' end to prevent extension by AmpliTaq Gold DNA polymerase (Perkin Elmer, Foster City, CA, USA). The level of expression of glyceraldehyde phosphate dehydrogenase (GAPDH) was measured as an internal standard, and the relative expression level of HB-EGF was estimated by comparison with the internal standard. The primer and probe sequences are listed in Table 1.

\begin{tabular}{|c|c|}
\hline Age (years, mean) & 63.5 \\
\hline \multicolumn{2}{|l|}{ Side $(\mathrm{N})$} \\
\hline right/left & $7 / 9$ \\
\hline \multicolumn{2}{|l|}{ Growing pattern $(\mathrm{N})$} \\
\hline expansive/intermediate & $14 / 2$ \\
\hline \multicolumn{2}{|l|}{ Structure(N) } \\
\hline alveolar/solid/cystic & $15 / 0 / 1$ \\
\hline \multicolumn{2}{|l|}{ Subtype (N) } \\
\hline clear cell/granular cell & $16 / 0$ \\
\hline \multicolumn{2}{|l|}{ Grade $(\mathrm{N})$} \\
\hline $\mathrm{G} 1 / \mathrm{G} 2 / \mathrm{G} 3$ & $7 / 9 / 0$ \\
\hline \multicolumn{2}{|l|}{$\operatorname{INF}(\mathrm{N})$} \\
\hline$\alpha / \beta$ & $12 / 4$ \\
\hline \multicolumn{2}{|l|}{$\mathrm{pT}(\mathrm{N})$} \\
\hline $\mathrm{T} 1 / \mathrm{T} 2 / \mathrm{T} 3 \mathrm{a} / \mathrm{T} 3 \mathrm{~b}$ & $1 / 12 / 2 / 1$ \\
\hline \multicolumn{2}{|l|}{$\mathrm{pV}(\mathrm{N})$} \\
\hline V0/V1a/V1b & $11 / 4 / 1$ \\
\hline $\mathrm{ECl}(\mathrm{N})$ & \\
\hline
\end{tabular}

\begin{tabular}{|l|l|}
\hline$+/$ - & \multicolumn{1}{|l|}{$3 / 13$} \\
\hline HB-EGF mRNA (RCC) (N) & $10 / 6$ \\
\hline positive/negative & $13 / 3$ \\
\hline HB-EGF mRNA (Normal) (N) & \multicolumn{2}{|l|}{} \\
\hline positive/negative & \multicolumn{2}{|l|}{} \\
\hline INF: Infiltrative Growth; ECI: Extra capsular Invasion
\end{tabular}

Table 1: Characteristics of the patients in the study

\section{Preparation of the standard template for the real-time quantitative polymerase chain reaction (PCR)}

After amplication of the HB-EGF target template by PCR, the PCR product was purified using SUPRECTM-02 (Takara, Biomedicals, Kyoto, Japan). The DNA concentration of the purified product was determined by measuring A260 and A280 using Genequant (Pharmacia, Dubendorf, Switzerland). The diluted DNA solution was used as a standard template.

\section{Synthesis of cDNA}

To produce cDNA from total RNA, reverse transcription (RT) was carried out using Ready-To-Go You-Prime-First Strand Beads (Pharmacia Biotech K.K., Tokyo). After the total RNA was incubated at 37 for 1 hour, the RT reaction conditions were incubated at 30 for 10 minutes, 37 for 60 minutes, and 99 for 5 minutes. The cDNA was analyzed immediately or stored at -20 until use.

\section{Real-time quantitative polymerase chain reaction}

Real-time quantitative PCR was performed by using the TaqMan probe and TaqMan PCR Core Reagents Kit (PE Applied Biosystems, Perkin Elmer Japan, Chiba) using an ABI PRISM 7700 Sequence Detector (PE Applied Biosystems, Perkin Elmer Japan) [11,12]. PCR was carried out in 96 sample tubes per assay ( $25 \mu \mathrm{l}$ per tube) in a reaction buffer containing 10×TaqMan Buffer A, $25 \mathrm{mM} \mathrm{MgCl} 2,10$ $\mathrm{mM} \mathrm{dA} / \mathrm{dC} / \mathrm{dG} / \mathrm{dUTP}, 20 \mu \mathrm{M}$ primers (forward and reverse), $0.125 \mathrm{U}$ of AmpliTaq Gold per reaction, $0.25 \mathrm{U}$ of AmpErase per reaction, and $5 \mu \mathrm{L}$ of cDNA sample. The PCR conditions were 50 for 2 minutes, and 95 for 10 minutes. PCR consisted of 15 seconds of denaturation at 95, and 1 minute of annealing and extension at 59 for 50 cycles. The TaqMan probe consisted of an oligonucleotide with a 5'-reporter dye and a downstream 3 '-quencher dye. The fluorescent reporter dye FAM was covalently linked to the 5 '-end of the oligonucleotide. This reporter dye is quenched by TAMRA, typically located at the 3'-end of the oligonucleotide. Fluorescence quenching depends on the spatial proximity of the reporter and quencher dyes.

\section{The analysis of the real-time quantitative polymerase chain reaction}

The real-time quantitative polymerase chain reaction was analyzed using the computer software Sequence Detector (PE Applied Biosystems, Perkin Elmer Japan). To determine the copy number of GAPDH mRNA, TaqMan GAPDH Control Reagents (PE Applied Biosystems, Perkin Elmer Japan) were used according to the manufacturer's instructions [13]. The amount of HB-EGF cDNA was normalized to that of GAPDH cDNA produced from the same volume 
Citation: Masuda H, Fukabori Y, Nakano K, Kobayashi M, Yamanaka H (2015) Expression of Heparin-binding Epidermal Growth Factor-like Growth Factor (HB-EGF) in Human Renal Cell Carcinoma . J Integr Oncol 5: 171. doi:10.4172/2329-6771.1000171

Page 3 of 4

of RNA sample. We evaluated the expression level of HB-EGF mRNA in normal and tumor tissues of the same case.

\section{Statistical analysis}

The possible associations between the level of HB-EFG mRNA clinical and pathological factors of renal cell carcinoma were analyzed using StatView J 4.02 software (Abacus Concepts, Berkley, CA, USA). Data were shown as the mean \pm S.E. The rank difference correlation test of Spearman was used for analyzing age and diameter. MannWhitney's U-test was used for analyzing the pathological factors. Histopathologic diagnosis in this study was made by one pathologist according to the General Rules for Clinical and Pathologic Studies on Renal Cancer edited by the Japanese Urological Association and the Japanese Society of Pathology [14]. A probability of $<0.05$ was considered significant. The necessary steps were taken to ensure our institutionethical policies for retrospective research projects were satisfied.

\section{Results}

The clinical features of the 16 patients and the pathological findings of 16 RCCs were shown in Table 2. All cases were clear cell type RCC. Negative expression of the HB-EGF gene in RCC was detected in 2 out of 7 (29\%) grade 1 and in 4 out of $9(44 \%)$ grade 2 cases. Thus, there was a lack of expression of the HB-EGF gene in about $38 \%$ of lower grade cancers. Moreover, with regard to the pattern of histological invasion, a lack of detectable expression of the HB-EGF gene was found in 3 out of 12 (25\%) INFatype and in 3 out of 4 (75\%) INF $\beta$ type tumors.

\begin{tabular}{|l|l|l|l|}
\hline & Target Gene & Primer/Probe & Sequence \\
\hline HB-EGF & HB-EGF & Primer sense & CACACCAAACAAGGAGGAGCAC \\
\hline & & Primer antisense & AAACATGAGAAGCCCCACGAT \\
\hline internal & GAPDH & TaqMan probe & CCATGTCTTCGGAAATACAAGGACTTCTGC \\
\hline standard & & Primer sense & CTTCACCACCATGGAGAAGGC \\
\hline & & TaqMan probe & GGCATGGACTGTGGTCATGAG \\
\hline
\end{tabular}

Table 2: Primer and TaqMan probe of HB-EGF and GAPDH

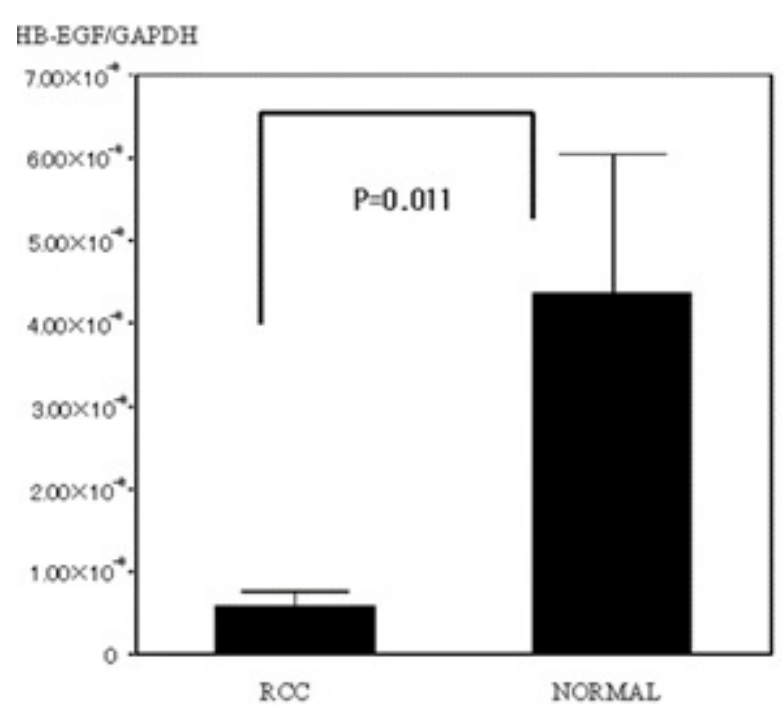

Figure 1: The expression of HB-EGF/GAPDH in renal cell carcinoma and normal tissues

Real-time PCR is a novel method for the quantitation of cDNA content in the $5^{\prime}$ nuclease assay. The threshold cycle $(\mathrm{Ct})$, defined as the point at which the amplification plot crosses the PCR baseline, decreases logarithmically with the input cDNA copy number. The $\mathrm{Ct}$ of each cDNA sample extracted from normal and tumor kidney tissues was within the linear phase, so that $\mathrm{Ct}$ values could be used as a quantitative measurement. The coefficients of correlation between $\mathrm{Ct}$ values and starting target copy number were 0.988 and 0.998 for the cDNA of HB-EGF and GAPDH, respectively.

The expression of HB-EGF mRNA was observed in $82 \%$ (13/16) of the normal tissues and $63 \%(10 / 16)$ of the tumor tissues. The average relative expression levels (HB-EGF/GAPDH) in normal and tumor tissues were $4.35 \times 10-6 \pm 1.7 \times 10-6$ (Mean \pm S.E.) and $5.95 \times 10-7 \pm$ $1.68 \times 10-7$, respectively. The former was significantly higher than the latter $(\mathrm{p}=0.011)$ (Figure 1). No significant correlation was found between the expression of HB-EGF mRNA and clinical stage, pathological parameters or prognosis. The expression of HB-EGF mRNA was not significantly correlated with age $(\mathrm{p}=0.27)$ or diameter of the tumor $(\mathrm{p}=0.84)$.

\section{Discussion}

RCC is a cancer which arises from the proximal tubule epithelium and accounts for $3 \%$ of adult cancers. Since RCC often shows an unpredictable clinical course, it is important to identify prognostic factors for RCC. Although it has been considered that the pathological stage is the most powerful prognostic marker for patients with RCC, many investigations have been performed to search for new predictive markers for this tumor. Recent studies have shown that nuclear morphometry, cell proliferation analyses, the expression of cell adhesion molecules and proteases, and the expression and serum level of growth factors are possible new candidates for useful prognostic markers [15]. In recent years, the expression of various factors in RCC has been reported, and it has been suggested that these factors are valuable for predicting prognosis. 
Page 4 of 4

HB-EGF is expressed in heart, lung, kidney and other many organs [3]. However, Miyoshi et al. [8] reported that HB-EGF was almost not expressed in normal liver tissues but highly expressed in the tumor tissues of HCC. There have been a few reports concerning HB-EGF expression in urological diseases. Torring et al. [16] investigated the expression of the EGF system by quantitative reverse transcriptionpolymerase chain reaction (RT-PCR) and enzyme-linked immunosorbent assay (ELISA) in a normal prostate epithelial cell line, and in an androgen sensitive-(LNCaP), and in an androgenindependent (DU145 and PC3) prostate cancer cell lines. They detected the increased expression of HB-EGF in androgenindependent prostate cancer cell lines. They suggested that this fact could reflect a mechanism to escape androgen dependence in prostate cancer. Freeman et al. [17] reported a study of immunolocalization of HB-EGF which revealed that prostate cancer cells, normal glandular epithelial cells, undifferentiated fibroblasts, and inflammatory cells did not react with anti-HB-EGF. However, interstitial and vascular smooth muscle cells were highly reactive, indicating that the smooth muscle compartments were the major sites of the synthesis and localization of HB-EGF within the prostate. They suggested that HB-EGF might be one of the stromal mediators of prostate cancer growth. Thus, the expression of HB-EGF may be involved in the progression and multiplication of prostatic cancer. We investigated the expression of HB-EGF mRNA in the tumor tissue and normal tissue in RCC, which was suggested to be of proximal tubule derivation, in order to clarify whether HB-EGF was related to the transition of normal cells to cancer cells.

The expression of HB-EGF was significantly lower in RCC tumor tissues than in normal tissues. We also found no association between any clinical factors and the expression of HB-EGF mRNA in normal or tumor tissues of RCC patients. In contrast to previous findings with HCC, our findings did not indicate that HB-EGF promoted the multiplication of RCC. The loss of HB-EGF expression was already found in a lower grade of RCC at a ratio of $38 \%$, and in higher grade cancer, there was a higher rate of loss of HB-EGF. A more-malignant infiltration pattern also was associated with a higher rate of loss of HBEGF expression. It has been reported that the differentiation of androgen-dependent prostatic cancer is influenced by the autocrine effects of the epidermal growth factor family [16]. A differentiationinducing effect of HB-EGF on renal epithelial cells has also been reported [18]. We found that the rate of negative expression of HBEGF was higher in RCC with more malignant progression. The normal regulation of the expression of HB-EGF mRNA might thus be disrupted in malignant progression.

The limitations of our present study included the retrospective design and small sample size. Because these data were not recently, we could not evaluate the existence of patient survival during the long period.

In conclusion, our results indicated that it was unlikely that HBEGF might play a role in determining the aggressiveness or clinical features in RCC. However, the decreased expression of HB-EGF mRNA in RCC tissues indicates that tumorigenesis of RCC may disrupt the normal regulatory system of HB-EGF.

\section{Funding}

No funding.

\section{Competing interests}

The authors declare no conflict of interest.

\section{References}

1. Higashiyama S, Abraham JA, Miller J, Fiddes JC, Klagsbrun M (1991) A heparin-binding growth factor secreted by macrophage-like cells that is related to EGF. Science 251: 936-939.

2. Higashiyama S, Lau K, Besner GE, Abraham JA, Klagsbrun M (1992) Structure of heparin-binding EGF-like growth factor. Multiple forms, primary structure, and glycosylation of the mature protein. J Biol Chem 267: 6205-6212.

3. Abraham JA, Damm D, Bajardi A, Miller J, Klagsbrun M, et al. (1993) Heparin-binding EGF-like growth factor: characterization of rat and mouse cDNA clones, protein domain conservation across species, and transcript expression in tissues. Biochem Biophys Res Commun 190: 125-133.

4. Nakagawa T, Hayase Y, Sasahara M, Haneda M, Kikkawa R, et al. (1997) Distribution of heparin-binding EGF-like growth factor protein and mRNA in the normal rat kidneys. Kidney Int 51: 1774-1779.

5. Raab G, Klagsbrun M (1997) Heparin-binding EGF-like growth factor. Biochim Biophys Acta 1333: F179-199.

6. Mitamura T, Higashiyama S, Taniguchi N, Klagsbrun M, Mekada E (1995) Diphtheria toxin binds to the epidermal growth factor (EGF)-like domain of human heparin-binding EGF-like growth factor/diphtheria toxin receptor and inhibits specifically its mitogenic activity. J Biol Chem 270: 1015-1019.

7. Duque JL, Adam RM, Mullen JS, Lin J, Richie JP, et al. (2001) Heparinbinding epidermal growth factor-like growth factor is an autocrine mediator of human prostate stromal cell growth in vitro. J Urol 165 : 284-288.

8. Miyoshi E, Higashiyama S, Nakagawa T, Suzuki K, Horimoto M, et al. (1996) High expression of heparin-binding EGF-like growth factor in rat hepatocarcinogenesis. Int J Cancer 68: 215-218.

9. Fink L, Seeger W, Ermert L, Hänze J, Stahl U, et al. (1998) Real-time quantitative RT-PCR after laser-assisted cell picking. Nat Med 4: 1329-1333.

10. Shimokawa T, Kato M, Ezaki O, Hashimoto S (1998) Transcriptional regulation of muscle-specific genes during myoblast differentiation. Biochem Biophys Res Commun 246: 287-292.

11. Heid CA, Stevens J, Livak KJ, Williams PM (1996) Real time quantitative PCR. Genome Res 6: 986-994.

12. Gibson UE, Heid CA, Williams PM (1996) A novel method for real time quantitative RT-PCR. Genome Res 6: 995-1001.

13. Perkin-Elmer (1996) Applied Bio systems in ABI PRISM 7700 Sequence Detector User's Manual.

14. Japanese Urological Association and the Japanese Society of Pathology (2011) General Rules for Clinical and Pathological Studies on Renal Cancer.

15. Shimazui $\mathrm{T}$ (1998) Recent progress of prognostic factors in renal cell carcinoma. Gan To Kagaku Ryoho 25: 2049-2054.

16. Tørring N, Jørgensen PE, Sørensen BS, Nexø E (2000) Increased expression of heparin binding EGF (HB-EGF), amphiregulin, TGF alpha and epiregulin in androgen-independent prostate cancer cell lines. Anticancer Res 20: 91-95.

17. Freeman MR, Paul S, Kaefer M, Ishikawa M, Adam RM, et al. (1998) Heparin-binding EGF-like growth factor in the human prostate: synthesis predominantly by interstitial and vascular smooth muscle cells and action as a carcinoma cell mitogen. J Cell Biochem 68: 328-338.

18. Takemura T, Hino S, Okada M, Murata Y, Yanagida H, et al. (2002) Role of membrane-bound heparin-binding epidermal growth factor-like growth factor (HB-EGF) in renal epithelial cell branching. Kidney Int 61: 1968-1979. 